\title{
Exactly solvable orbifold models and subfactors
}

\author{
YASUYUKI KAWAHIGASHI* \\ Department of Mathematics \\ University of California, Berkeley, CA 94720
}

\section{$\S 1$ Introduction}

Since the pioneering work of V. F. R. Jones [J], the theory of subfactors has had deep and unexpected relations to 3-dimensional topology, conformal field theory, quantum groups, etc. Here we present a relation between paragroup theory of Ocneanu on subfactors and exactly solvable lattice models in statistical mechanics, and in particular, show usefulness of the notion of orbifold lattice models in subfactor theory.

Classification of subfactors of the approximately finite dimensional (AFD) factor of type $\mathrm{II}_{1}$ is one of the most important and challenging problems in the theory of operator algebras. This is a study of inclusions of certain infinite dimensional simple algebras of bounded linear operators on a Hilbert space. The AFD type $\mathrm{II}_{1}$ factor, the operator algebra we work on, is the most natural infinite dimensional analogue of finite dimensional matrix algebras $M_{n}(\mathbf{C})$ in a sense. (As a general reference on operator algebras, see $[\mathrm{T}]$, and as a basic reference on subfactor theory, we cite [GHJ]. All the basic notions are found there.) Here, by "a subfactor $N \subset M$ " we mean that $N$ and $M$ are both AFD type $\mathrm{II}_{1}$ factor and $N$ is a subalgebra of $M$. V. Jones studied the Jones index $[\mathrm{J}]$, a real-valued invariant for the inclusion $N \subset M$, which measures the relative size of $M$ with respect to $N$, roughly speaking.

A classification approach based on higher relative commutants were studied by several people such as Jones, Ocneanu, Pimsner and Popa. In this approach, the classification problem can be divided into the following 3 steps.

(1) Prove that the subfactor can be approximated by certain increasing sequence of finite dimensional algebras called higher relative commutants.

(2) Characterize the higher relative commutants in an axiomatic way.

(3) Work on the axioms to classify higher relative commutants.

Part (1) is quite functional analytic, but parts (2) and (3) are rather algebraic and combinatorial. We are concerned mainly with (2) and (3) in this paper.

V. Jones noticed that some graph called "principal graph" appears naturally from the higher relative commutants. A. Ocneanu [O1] first claimed that Step (1) is possible in the case with certain finiteness condition called "finite depth," which means that the principal graph is finite. As to Step (2), he further obtained a complete combinatorial characterization of higher relative commutants for the finite depth case, found a new algebraic structure, and named it paragroup. It was known that if the Jones

*Miller Research Fellow

Permanent address:Department of Mathematics, Faculty of Science

University of Tokyo, Hongo, Tokyo, 113, JAPAN 
index is less than 4, then the principal graph must be one of the Coxeter-Dynkin diagrams $A_{n}, D_{n}, E_{6}, E_{7}, E_{8}$. Then Ocneanu announced a complete classification of these subfactors through his paragroup approach in [O1].

But unfortunately, details of Ocneanu's proofs have not appeared. As to Step (1) of the above, S. Popa published a complete proof [P2] with the finite depth condition and without assuming the so-called trivial relative commutant property $N^{\prime} \cap M=\mathbf{C}$. Furthermore, he announced an ultimate result along this line [P3] and full details are soon appearing. Thus, we now have a satisfactory theory about Step (1), and in this paper, we work on steps (2) and (3).

On step (2), A. Ocneanu has the following four different viewpoint for paragroups.

(a) "Quantized" Galois groups

(b) "Discrete" version of compact manifolds

(c) Exactly solvable lattice models without a spectral parameter

(d) Finite tensor categories giving topological invariants of 3-manifolds

Though we mainly work on the third viewpoint (c), we make a quick explanation on each aspect. The classical Galois theory deals with inclusions of a subfield in another field. Here we work on inclusions of an (infinite dimensional non-commutative) algebra in another. Passing to non-commutative settings is often referred (vaguely) as quantization.

In paragroup theory, we work on finite graphs and some structure on it called "connections." This can be regarded as a discrete version of connections in differential geometry, and an analogue of flatness of connections will play a key role.

In solvable lattice model theory, they have a notion of IRF (interaction around faces) models. They have a graph and certain structure called Boltzmann weights on it. This Boltzmann weight depends on a parameter called a spectral parameter, and satisfies certain axioms. Axioms for paragroup are very similar to those of IRF models without a spectral parameter. Indeed, we have the following correspondence table.

\begin{tabular}{|l|l|}
\hline Paragroups & IRF models \\
\hline \hline Connections & Boltzmann weights \\
\hline Unitarity & First inversion relations \\
\hline Commuting square conditions & Second inversion relations \\
\hline $\begin{array}{l}\text { Commuting square conditions } \\
\text { for higher relative commutants }\end{array}$ & Crossing symmetry \\
\hline Flatness of connections & Yang-Baxter equation plus something (?) \\
\hline
\end{tabular}

We show more details on this later. (See [Ba, DJMO, Ji] for more on lattice models.)

A. Ocneanu recently announced in [O4] that paragroups are in bijective correspondence to certain type of complex-valued topological invariants of 3-dimensional manifolds. This construction is a generalization of the Turaev-Viro invariant [TV].

In this paper, we first make a review of Ocneanu's paragroup theory, and then go into our idea of using orbifold models in paragroup settings. Roughly speaking, this idea is making a quotient of a paragroup for certain symmetry having a fixed point. In this way, we can solve some problems and give new constructions in subfactor theory. The 
notion of orbifold subfactors were studied through the joint work with D. E. Evans. The author thanks Professors D. Bisch, M. Choda, U. Haagerup, M. Izumi, V. F. R. Jones, T. Miwa, A. Ocneanu, M. Okado, S. Okamoto, S. Popa, H. Wenzl, and S. Yamagami for several useful comments and conversations during this work.

$\S 2$ Bijective correspondence between paragroups and finite depth subfactors

Although A. Ocneanu announced a combinatorial axiomatization of higher relative commutants as paragroups in [O1], full details of his proof have not appeared. But this characterization is now fully understood from his various lectures [O2, O3]. Because necessary arguments are scattered among the several literatures like [O1, O2, O3, P2, $\mathrm{Ka}$ ] some of which are not widely available, here we give an exposition on how to obtain the bijective correspondence between paragroups and finite depth subfactors.

We follow a formal string algebra approach of [O2] rather than $\mathrm{II}_{1}$ factor bimodule approach [O1, O3]. This has a disadvantage that meaning is less clear, but has an advantage that the method is entirely elementary and more general than the bimodule approach. (For example, formal approach based on connection also easily works for subfactors of Goodman-de la Harpe-Jones [GHJ]. See Remark 2.2.) For details of the $\mathrm{II}_{1}$ factor bimodule approach, also see $[\mathrm{Y}]$.

Let $N \subset M$ be a subfactor of the AFD factor of type $\mathrm{II}_{1}$ with finite index and finite depth. (In the rest of this paper, we mean by a subfactor only the above type of subfactors.) Repeating the downward basic construction, we choose a tunnel

$$
\cdots N_{3} \subset N_{2} \subset N_{1} \subset N \subset M
$$

(See $[J, G H J]$ for basic definitions.) Consider the following canonical commuting squares in the sense of Popa.

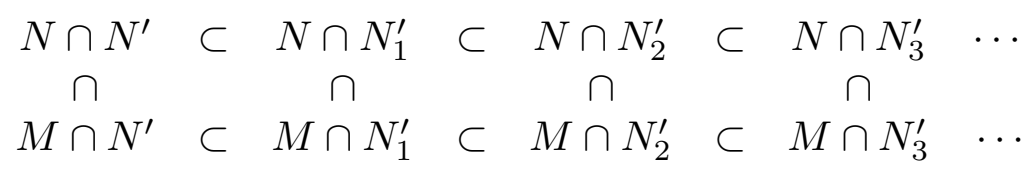

By Theorem of S. Popa [P2] (announced by A. Ocneanu), we may assume that each line above converges to $N$ and $M$ respectively by a certain choice of a tunnel. When we look at the Bratteli diagram of the sequence above, we find that there are four bipartite graphs $\mathcal{G}_{1}, \mathcal{G}_{2}, \mathcal{H}_{1}, \mathcal{H}_{2}$ connected as follows.

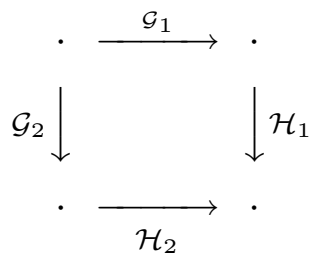

That is, we consider the following sequence of graphs and there is a distinguished vertex at the upper left corner of the diagram above so that all the paths starting from $*$ in 
the following diagram gives the above-mentioned Bratteli diagram.

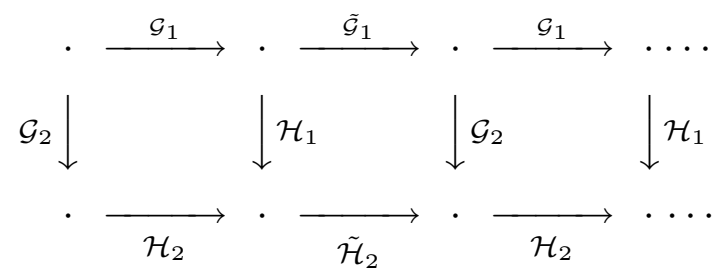

Here the notation ${ }^{\sim}$ means the reflection of the graph. Furthermore, $\mathcal{G}_{1}$ and $\mathcal{G}_{2}$ are the same (the principal graph), and $\mathcal{H}_{1}$ and $\mathcal{H}_{2}$ are the same (the dual principal graph). The ways $\mathcal{G}_{1}$ and $\mathcal{H}_{1}$ are connected and $\mathcal{G}_{2}$ and $\mathcal{H}_{2}$ are connected are the same. But the ways $\mathcal{G}_{1}$ and $\mathcal{G}_{2}$ are connected and $\mathcal{H}_{1}$ and $\mathcal{H}_{2}$ are connected may be non-trivial. This non-triviality is handled by the contragredient map in the sense of Ocneanu [O1]. (For these, see $[\mathrm{P} 2, \S 6]$, for example.)

Because we assume the finite depth condition, the Bratteli diagram above starts to have period 2 after finite stages. Let $k$ be the minimal even integer at which the commuting square

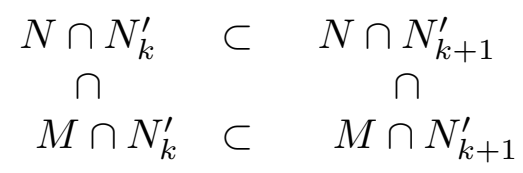

starts to be periodic. If we have inclusions of four finite dimensional $C^{*}$-algebras, this diagram is described by string algebras and connections as in [O1, O2, O3]. (String algebra description of an increasing system of finite dimensional $C^{*}$-algebra was introduced in [E1, E2] with the name of path algebra.) A full proof of this was given in [O2], and only elementary linear algebra is needed for the proof. Here by "connection", we mean an assignment of complex numbers to each square of the following form.

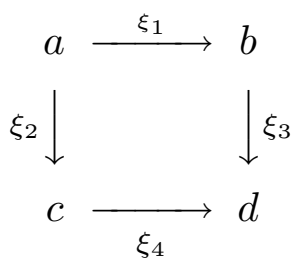

where $\xi_{1}, \xi_{2}, \xi_{3}, \xi_{4}$ are edges of $\mathcal{G}_{1}, \mathcal{G}_{2}, \mathcal{H}_{1}, \mathcal{H}_{2}$ respectively, and $a, b, c, d$ are vertices of the appropriate graphs. (Remember that each graph is bipartite and we identify even vertices of $\mathcal{G}_{1}$ and $\mathcal{G}_{2}$, and so on.) The connection is not uniquely determined from the four finite dimensional algebras, but it is unique up to certain equivalence relation discussed in [O1, O2]. Equivalent connections differ by so-called gauge choice freedom. This setting is quite similar to that of exactly solvable IRF (interaction around faces) lattice models [Ba, DJMO]. Usually in their setting, our four graphs are the same single one, which is sometimes oriented. They call above type of squares arising from the graph admissible, and their notion of Boltzmann weight corresponds to our notion of connection. (Further similarity between IRF models and paragroups will be discussed later, but we do not have a parameter corresponding to the spectral parameter in IRF models.) Rougly speaking, the connection gives identification of two basis on the algebra $M \cap N_{k+1}^{\prime}$ arising from the two inclusions $N \cap N_{k}^{\prime} \subset N \cap N_{k+1}^{\prime} \subset M \cap N_{k+1}^{\prime}$ and $N \cap N_{k}^{\prime} \subset M \cap N_{k}^{\prime} \subset M \cap N_{k+1}^{\prime}$. (Each inclusion gives an expression of a string 
algebra.) Because this identification must be a $*$-isomorphism, we have the following unitarity axiom.

$$
\begin{aligned}
& a \stackrel{\xi_{2}}{\longrightarrow} b \quad b \stackrel{\xi_{2}}{\longleftarrow} a
\end{aligned}
$$

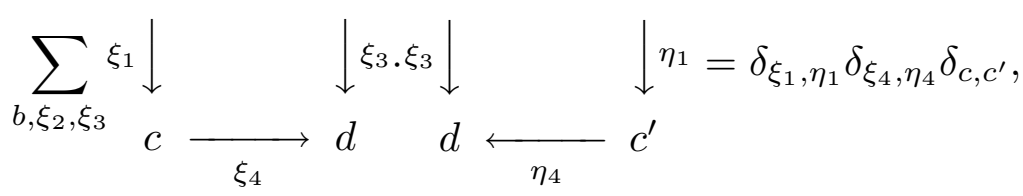

$$
\begin{aligned}
& a \stackrel{\xi_{2}}{\longrightarrow} b \quad b^{\prime} \stackrel{\eta_{2}}{\longleftarrow} a
\end{aligned}
$$

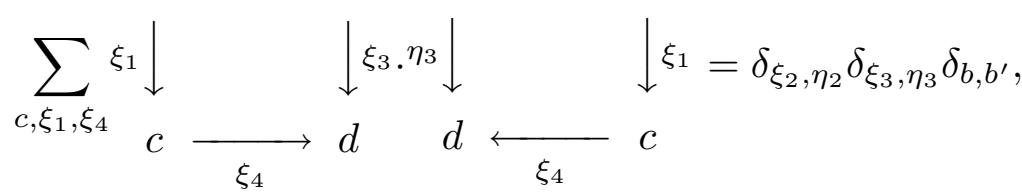

Here we used the following conventions.

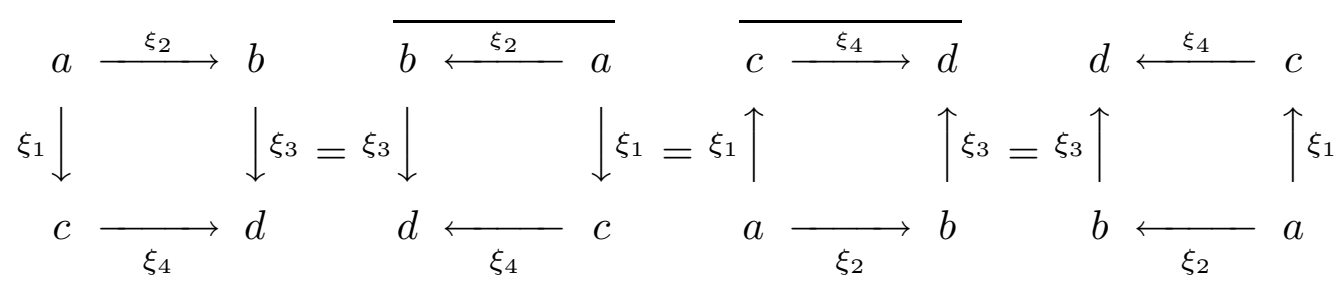

In IRF model theory, they call the corresponding axiom unitarity or the first inversion relations $[\mathrm{Ba}]$.

Now our four algebras give not just inclusions but a commuting square. This gives a certain condition on the connection. Because we can write down the trace and the conditional expectation explicitly as in [O1, O2, O3], we can write down the commuting square condition in terms of the connection, and by comparing the coefficients in the both hand sides, we get the following.

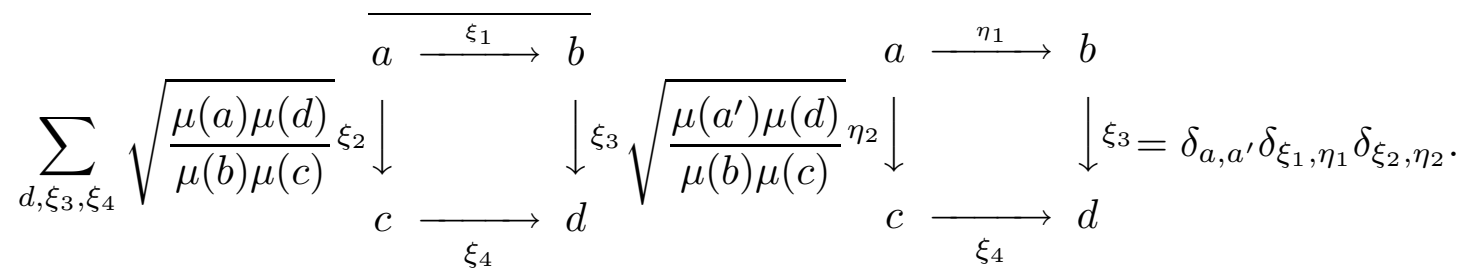

Here the notation $\mu(\cdot)$ means the entry of the Perron-Frobenius eigenvector. A proof for this is given in $[\mathrm{O} 2, \mathrm{Sc}]$ and quite elementary. This formula corresponds to the second inversion relations in the IRF models [Ba], so we also call this the second inversion relations. Then for our admissible squares, we define the following values for other types of squares. 


$$
\begin{aligned}
& d \stackrel{\tilde{\xi}_{4}}{\longrightarrow} c \quad a \stackrel{\xi_{1}}{\longrightarrow} b \\
& \tilde{\xi}_{3} \downarrow \quad \downarrow \tilde{\xi}_{2}=\xi_{2} \downarrow \quad \downarrow \xi_{3} \\
& b \underset{\tilde{\xi}_{1}}{\longrightarrow} a \quad c \underset{\xi_{4}}{\longrightarrow} d
\end{aligned}
$$

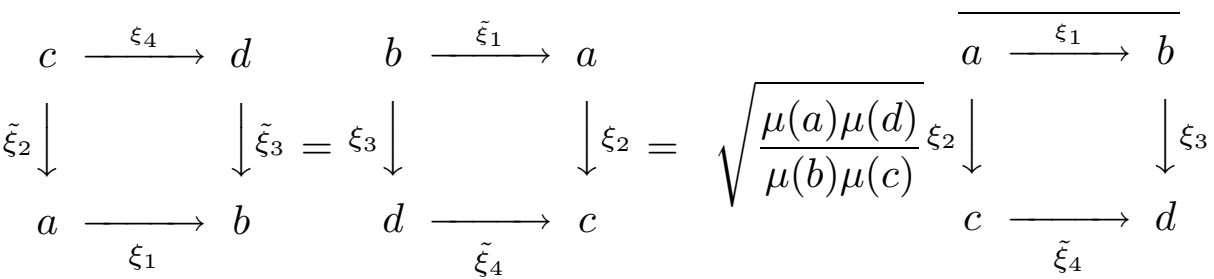

where the notation $\tilde{\xi}_{j}$ means the edge with its orientation reversed. Then by the second inversion relation, the above also satisfy unitarity. The definitions above are an analogue of crossing symmetry in IRF model theory [Ba]. So we also call these crossing symmetry. We call the above assignment of complex numbers to all the admissible squares (biunitary) connection. (The name "biunitary" connection of Ocneanu comes from that there are two kinds of unitarity: first and second inversion relations.) In this way, we obtain a connection from a subfactor. In short, commuting squares arising as the higher relative commutants correspond to crossing symmetry and more general commuting squares correspond to the second inversion relations in IRF model theory. Note that the renormalization convention for crossing symmetry here is slightly different from that in [O1] and the same as in [O3, EK, IK, Ka].

A connection arising from a subfactor satisfies another important axiom called flatness. In order to explain it, we first show how to construct a subfactor from a biunitary connection on two graphs $\mathcal{G}, \mathcal{H}$ with the same Perron-Frobenius eigenvalues.

We have the distinguished point $*$ among the even vertices of $\mathcal{G}$, which corresponds to the starting vertex of the Bratteli diagram for $N_{k}^{\prime} \cap N$. Then, we can construct a double sequence of string algebras starting from $*$ :

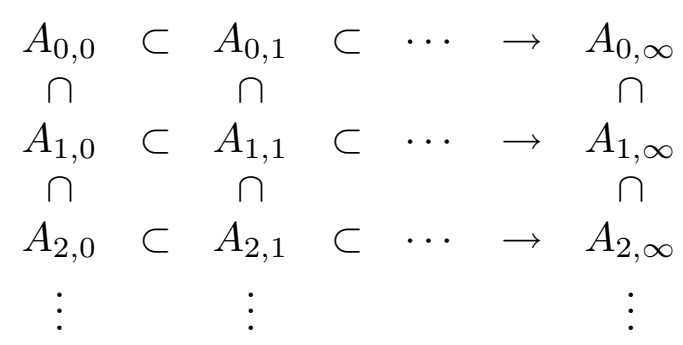

Identification for different expressions of strings are again given by the connection. See [O1, page 128] or [O3, II.1-2] for more details. A trace compatible with the embeddings above can be defined with the Perron-Frobenius eigenvector entries, and $A_{0, \infty}$, etc., are the GNS-completions with respect to this trace. (See [O1, page 129] or [O3, II.1] for this trace.) Then the inclusion $A_{0, \infty} \subset A_{1, \infty}$ is the string model subfactor of Ocneanu. We claim that if the connection arises from a subfactor $N \subset M$ in the above way, this subfactor $A_{0, \infty} \subset A_{1, \infty}$ is conjugate to $N \subset M$. 
This is proved as follows. First note that we have a sequence of the Jones projections $\left\{e_{-n}\right\}$ with $N_{n}=N_{n-1} \cap\left\{e_{-n+1}\right\}^{\prime}$. We identify the increasing sequence of the string algebra

$$
A_{0,0} \subset A_{0,1} \subset \cdots \subset A_{0, k} \subset A_{0, k+1} \subset A_{1, k+1}
$$

with the sequence

$$
N \cap N^{\prime} \subset N \cap N_{1}^{\prime} \subset \cdots \subset N \cap N_{k}^{\prime} \subset N \cap N_{k+1}^{\prime} \subset M \cap N_{k+1}^{\prime},
$$

where $k$ is as in the definition of the connection. Next we make identification of the following two sequences

$$
\begin{gathered}
A_{0, k+1} \subset A_{0, k+2} \subset A_{0, k+3} \subset \cdots \\
N \cap N_{k+1}^{\prime} \subset N \cap N_{k+2}^{\prime} \subset N \cap N_{k+3}^{\prime} \subset \cdots
\end{gathered}
$$

so that the Jones projections $\left\{e_{-n}\right\}, n \geq k+1$, have the expressions

$$
e_{-n}=\sum_{\substack{|\alpha|=n-1 \\|v|=|w|=1}} \frac{\mu(r(v))^{1 / 2} \mu(r(w))^{1 / 2}}{\beta \mu(r(\alpha))}(\alpha \cdot v \cdot \tilde{v}, \alpha \cdot w \cdot \tilde{w}),
$$

where $\beta$ is the Perron-Frobenius eigenvalue of the graph (which is equal to the square root of $[M: N]), \alpha$ is any horizontal path from $*$ of $\mathcal{G}$, and $v, w$ are chosen so that the compositions are possible, and $|\cdot|$ denote the length of a path. This is possible because the expressions above satisfy all the required properties for the Jones projections. The expressions above for the Jones projections are due to Ocneanu [O3, II.3] and Sunder $[\mathrm{Su}]$. We also identify two sequences

$$
\begin{gathered}
A_{1, k+1} \subset A_{1, k+2} \subset A_{1, k+3} \subset \cdots \\
M \cap N_{k+1}^{\prime} \subset M \cap N_{k+2}^{\prime} \subset M \cap N_{k+3}^{\prime} \subset \cdots
\end{gathered}
$$

so that the Jones projections have the same expressions for the other graph $\mathcal{H}$. Then we claim that these two identifications are compatible with the connection. To see this, it is enough to check the Jones projections on the first horizontal line are transformed to the Jones projections on the second horizontal line by the connection, but this is valid as in [O3], which is the same as flatness of the Jones projections implied by the crossing symmetry. In this way, we get an isomorphism of $A_{1, \infty}$ onto $\vee_{n}\left(M \cap N_{n}^{\prime}\right)$ which carries $A_{0, \infty}$ onto $\vee_{n}\left(N \cap N_{n}^{\prime}\right)$. Because we assumed that the tunnel has the generating property, we are done.

Now we work on the double sequence of string algebras arising from a biunitary connection which may not come from a subfactor. We define the vertical Jones projections $\left\{e_{n}\right\}$ with $e_{n} \in A_{n-1,0}$ with the same expression as above for the vertical string algebra of $\mathcal{G}$ from $*$. Then the crossing symmetry again implies the flatness of the vertical Jones projections and we can conclude that

$$
A_{0, \infty} \subset A_{1, \infty} \subset A_{2, \infty} \subset A_{3, \infty} \subset \cdots
$$

is the Jones tower of the inclusion $A_{0, \infty} \in A_{1, \infty}$ by [PP, Proposition 1.2]. 
One of the main problems in subfactor theory is a computation of higher relative commutants $N^{\prime} \cap M_{n}$ for a given construction of a subfactor $N \subset M$. Suppose we have a subfactor $N=A_{0, \infty} \subset M=A_{1, \infty}$ as above constructed from a double sequence of string algebras with a connection on finite graphs which may not come from a subfactor. Then Ocneanu found that $N^{\prime} \cap M_{n}$ is always a subalgebra of $A_{n+1,0}$ and gave a nice combinatorial characterization for strings in $A_{n+1,0}$ to be in $N^{\prime} \cap M_{n}$ with his compactness argument [O3, II.6]. In many natural cases, we have equality $N^{\prime} \cap M_{n}=A_{n+1,0}$. Because we have two graphs $\mathcal{G}$ and $\mathcal{H}$, we can repeat the same construction with $\mathcal{G}$ and $\mathcal{H}$ interchanged. If we have equalities $N^{\prime} \cap M_{n}=A_{n+1,0}$ in the both cases, we say that the connection is flat. We now claim that a connection arising from a subfactor $N \subset M$ is flat.

We already know that $A_{0, \infty}=N, A_{1, \infty}=M$, and $N^{\prime} \cap M_{n} \subset A_{n+1,0}$. But both $N^{\prime} \cap M_{n}$ and $A_{n+1,0}$ are identified with the string algebra with length $n+1$ of $\mathcal{G}$ starting from $*$, so these two algebras have the same dimensions. With the inclusion above, we get $N^{\prime} \cap M_{n}=A_{n+1,0}$. Next we define $A_{1,-1}=\mathbf{C}$ and regard the sequence

$$
A_{1,-1} \subset A_{1,0} \subset A_{1,1} \subset A_{1,2} \subset \cdots
$$

as an increasing sequence of string algebras of $\mathcal{H}$ starting from $*$ of $\mathcal{H}$. This is possible because we have the same number of edges from $*$ of $\mathcal{G}$ and $*$ of $\mathcal{H}$ and each pair of vertices of $\mathcal{G}$ and $\mathcal{H}$ is identified. Furthermore, we construct a vertical increasing sequence of string algebras

$$
A_{1,-1} \subset A_{2,-1} \subset A_{3,-1} \subset A_{4,-1} \subset \cdots
$$

as an increasing sequence of string algebras of $\mathcal{H}$ starting from $*$ of $\mathcal{H}$. Then we embed $A_{n,-1}$ into $A_{n, 0}$ using the graph $\mathcal{G}$ or $\mathcal{H}$ according to parity of $n$. In this way, we extend the double sequence to the following form.

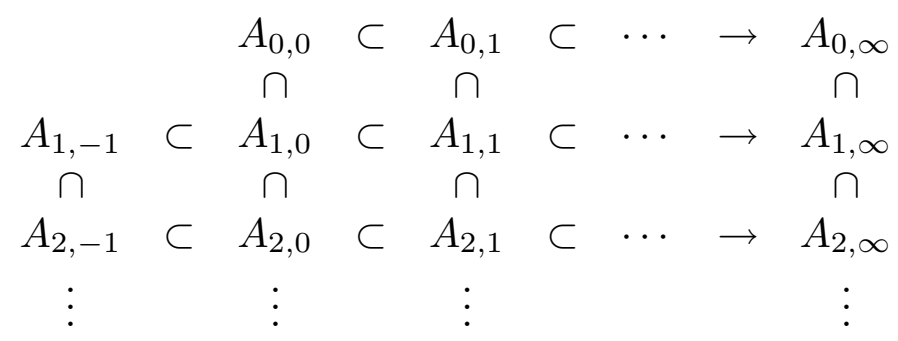

Now the same kind of argument as above gives $M^{\prime} \cap M_{n}=A_{n+1,-1}$, which means flatness as desired.

By the above, we know that if we make a flat connection from a subfactor and then make a subfactor from the flat connection, we get the same subfactor back. Conversely, we will prove that if we make a subfactor from a flat connection and then make a flat connection from the subfator, we get the same flat connection back. So suppose we have a double sequence of string algebras arising from a flat connection. By similar method 
to the above, we can extend the double sequence to the following form.

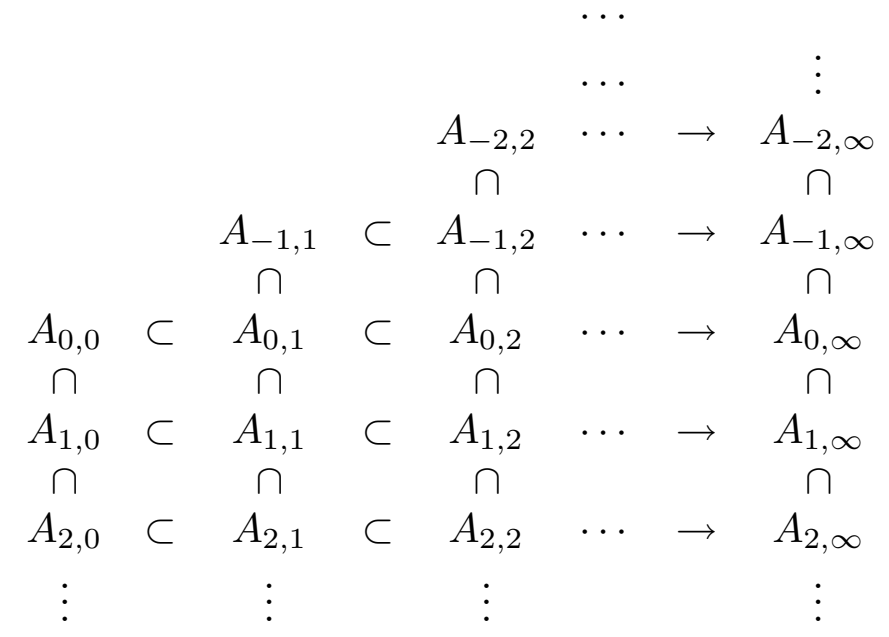

That is, we have $A_{m, n}$, for all $m, n$ with $-m \leq n, 0 \leq n$. By the same kind of argument as above, we can prove that

$$
A_{0, \infty} \supset A_{-1, \infty} \supset A_{-2, \infty} \supset \cdots
$$

is a tunnel. Flatness now implies that $A_{0, n}=A_{-n, \infty}^{\prime} \cap A_{0, \infty}$ and $A_{1, n}=A_{-n, \infty}^{\prime} \cap A_{1, \infty}$. In this way, we get the conclusion. (By this arguments, we also proved that the tunnel above has the generating property.)

Note that identification of flat connections are given by gauge choice freedom and graph isomorphism as in [O1, O2, O3]. Thus, we have established the bijective correspondence between conjugacy classes of subfactors and equivalence classes of flat connections. Flatness does not have a direct analogue in IRF model theory unlike the first and second inversion relations, but in some "good" cases, the flatness is obtained from the Yang-Baxter equation in the IRF model theory. This will be discussed in $\S 4$ in connection with Hecke algebra subfactors of Wenzl.

The next topic is how to check flatness for a given biunitary connection. We work only on the equality $N^{\prime} \cap M_{n}=A_{n+1,0}$ because the other is handled in the same way. First note that this can be stated as $x y=y x$ for $x \in A_{n, 0}, y \in A_{0, m}$. This form is conceptually simple. But the difficulty is that we have infinitely many strings for which we have to check commutativity. Because we assume that the both graphs $\mathcal{G}, \mathcal{H}$ are finite, the increasing sequence of the string algebra is generated by the Jones projections and some finitely many elements. Flatness is automatically satisfied for the Jones projections, so it is enough to check commutativity only for finitely many elements. In this way, checking flatness is reduced to finitely many computations. Furthermore, the following theorem gives computational methods to check flatness.

Theorem 2.1. The following conditions are equivalent.

(1) In the double sequence of string algebras, any two elements $x \in A_{\infty, 0}$, the vertical string algebra, and $y \in A_{0, \infty}$, the horizontal string algebra, commute. 
(2) For each vertical string $\rho=\left(\rho_{+}, \rho_{-}\right) \in A_{k, 0}$, we get

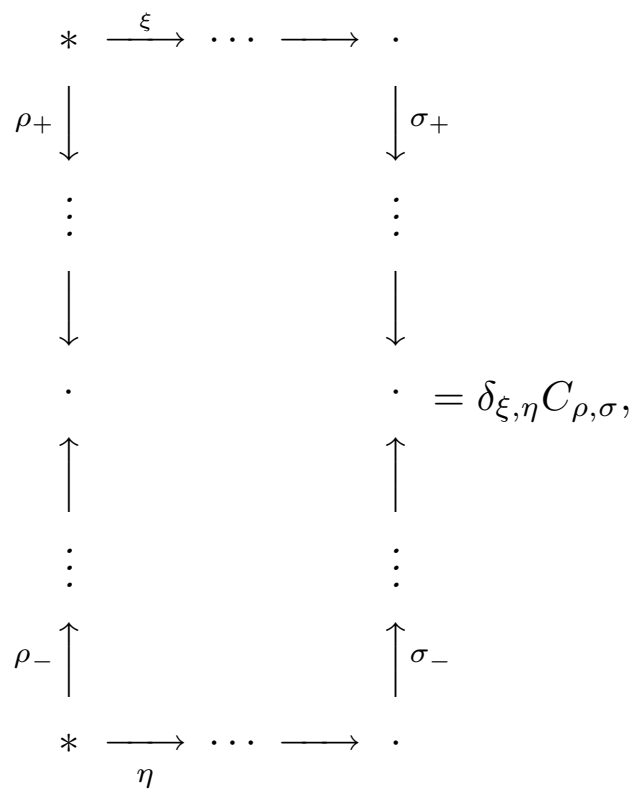

where $C_{\rho, \sigma} \in \mathbf{C}$ depends only on $\rho, \sigma=\left(\sigma_{+}, \sigma_{-}\right)$.

(3) For any horizontal paths $\xi_{+}, \xi_{-}$and vertical paths $\eta_{+}, \eta_{-}$with all the sources and ranges equal to $*$, we get

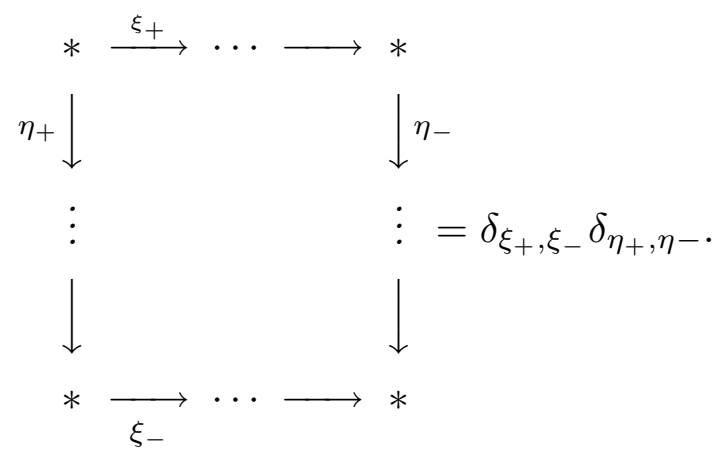

In the theorem above, the meaning of large diagrams are as follows. We consider all the possible ways of filling the large diagram with admissible squares. Each such a choice is called a configuration. We multiply the connection values of all the admissible squares in each configuration and sum them over all the configurations. This is the value assigned to the large diagram above, and we mean this value by the diagram. This is an analogue of a partition function in the IRF model theory.

Condition (3) above was used as definition of flatness by Ocneanu [O1], and equivalence to (2) was claimed in [O1] without proof. The name "flat connection" comes from a fact that these conditions are analogues of conditions for flat connections in differential geometry. A proof for the theorem above was given in [Ka]. Again, in order to check flatness for a given connection, we need to verify (2) or (3) only for finitely many diagrams. Biunitarity is a local axiom in the sense that we can check it just by looking at a part of the graph for each equality, but this flatness axiom is a global axiom, and in general, very hard to verify. For example, in the case of index less than 4, the principal graph must be one of the Dynkin diagrams of type $A, D, E$ and the dual principal graph 
must be the same. Then it is not so difficult to get all the connections on them [O1, O3, Ka]. That is, there is a unique connection on each of $A_{n}, D_{n}$, and there are two connections on each of $E_{6}, E_{7}, E_{8}$. So classification of subfactors with index less that 4 is reduced to flatness problem of these connections, which requires deeper considerations.

Remark 2.2. A series of subfactors was constructed by [GHJ] for the Coxeter-Dynkin diagrams, and S. Okamoto [Ok] computed the principal graphs for them. This result follows very easily from the above general settings of a double sequence of string algebras with a connection which does not come from a subfactor. By [GHJ], we know that the subfactor arises from the double sequence of string algebras with a biunitary connection on the following type of diagram.

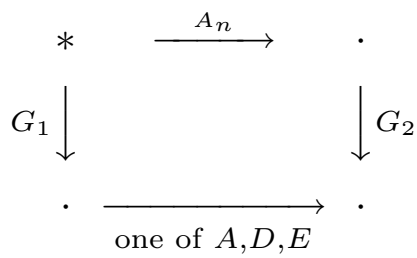

where $G_{1}$ and $G_{2}$ are some graphs with the same Perron-Frobenius eigenvalues, which can be computed easily. Then it is easy to see the method above of computation of the principal graph also works for this connection. The horizontal string algebra is now generated by the Jones projections, so flatness of this connection immediately follows from the flatness of the Jones projections. (That is, each vertical string commutes with each horizontal string.) Thus, the vertical graph $G_{1}$ is the principal graph of the subfactor. (Both Ocneanu and Okamoto said to the author that they had known this simple method.)

It is also possible to compute the connection explicitly. For example, $[\mathrm{R}]$ has a table of connection values for the subfactor with index $3+\sqrt{3}$ arising from $E_{6}$ and [DZ] has more. (Roche calls it a cell system and used it for a different purpose.)

The graph $\mathcal{G}_{2}$ in the diagram above is not the "dual" principal graph in general, and it is very difficult to compute the dual principal graph from Ocneanu's general machinery. But in some cases, purely combinatorial arguments determine the dual principal graph. For example, in the case with index $3+\sqrt{3}$, the dual principal graph must be the same as $G_{1}$, Okamoto's graph [Ok], as conjectured at the end of [Ok]. (But Haagerup [H] proved a much stronger result. That is, if a finite depth subfactor has index $3+\sqrt{3}$, then its principal graph must be Okamoto's.)

If we choose the graph $A_{n}$ from $A-D-E$ 's in the construction above, then we get the index values $\left(\sin ^{2} k \pi / N\right) /\left(\sin ^{2} \pi / N\right)$, same as Wenzl's index values for his Hecke algebra subfactors [We]. But Ocneanu's machinery tells that the construction here gives a subfactor arising from basic construction of subfactors of type $A$. This corresponds to Wenzl's remark in [We, page 360] and is different from his Hecke algebra subfactors. Principal graphs of these subfactors were computed by Izumi [I1, Figures 5, 7] and principal graphs of the Hecke algebra subfactors were computed by unpublished work of Wenzl and [EK].

For the diagrams $D_{2 n}, E_{6}, E_{8}$, for which flat connections exist, each diagram has the distinguished vertex $*$ which is characterized as having the smallest entry of the PerronFrobenius eigenvector. If we choose a vertex different from $*$ in the construction above, 
then the resulting subfactor contains a non-trivial intermediate subfactor corresponding to the string algebra starting from $*$. In this sense, it is not a "simple" object.

$\S 3$ Orbifold construction I: $*$ is fixed.

Now we come to the main topic of this paper: orbifold subfactors. This is an analogue of orbifold models in IRF model theory $[\mathrm{Ko}, \mathrm{FG}]$ and the idea is quite simple. That is, if we have a certain symmetry of a paragroup, we can make a quotient of the paragroup by the symmetry and produce a new paragroup. In operator algebraic setting, this means that we take a fixed point algebra of the string algebra by the automorphism with finite order arising from the paragroup symmetry. Recall that in $C^{*}$-algebra theory, an idea of non-commutative orbifold has been also successful [BEEK1-3, BEK, BK].

First we point out that we have to consider two type of symmetries of a paragroup separately. The first case is symmetries fixing the distinguished vertex $*$ of the paragroup, and the second is symmetries moving $*$. Though the second case is more interesting, the first case is easier, so we work on the first case in this section. This type of symmetry is also related to Loi's work [L1], [L2, Lemma 4.2].

In order to keep arguments simple, we assume that the two graphs of the paragroup have no multiple edges and let $\sigma$ be a non-trivial automorphism of the graphs which keeps the connection invariant:

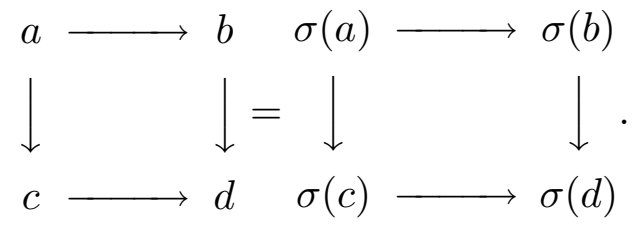

(Because we have no multiple edges, we drop labeling of edges.)

Then this $\sigma$ defines an automorphism of the string algebra, and this is well-defined on the double sequence of string algebras because of the invariance of the connection. We denote this automorphism by $\sigma$ again and take fixed point algebra $A_{m, n}^{\sigma}$ of each $A_{m, n}$ in the double sequence. Then $A_{m, n}^{\sigma}$ gives a double sequence of commuting squares, and the Jones projections are invariant under $\sigma$, so we have another double sequence with each horizontal line defining the Jones tower. Next we would like to check flatness of this system, but this is automatically satisfied because each algebra in the double sequence in a subalgebra of an algebra in the original double sequence where we have flatness and then commutativity is trivial. In this way, we get another subfactor with the same index and a different principal graph. (It is easy to see that the index is value is kept in this procedure because the Jones projections are invariant under the symmetry. We can also appeal to [GHJ] or [Wa, page 227].) The new principal graph is an "orbifold" graph of the original principal graph. (M. Choda worked on this kind of graph problem in more abstract settings in [Ch].)

A simple example is as follows. An abelian group $\mathbf{Z}_{n}$ is realized as a paragroup as follows. The two graphs $\mathcal{G}$ and $\mathcal{H}$ are the same and it has a single odd vertex $x$ and $n$ single edges with length 1 from $x$. Each even vertex of $\mathcal{G}$ corresponds to an element of 
$\mathbf{Z}_{n}$ and each of $\mathcal{H}$ to an element of $\hat{\mathbf{Z}}_{n}$. The distinguished vertex $*$ corresponds to 0 in $\mathbf{Z}_{n}$ and $\hat{\mathbf{Z}}_{n}$. The connection is given by

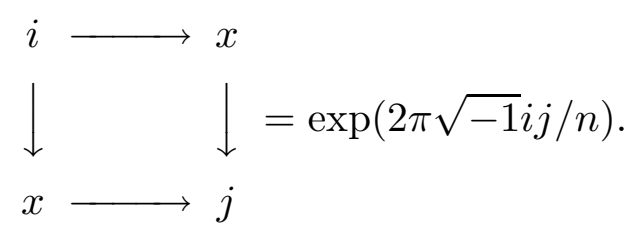

(The other types of admissible squares have the values determined by the crossing symmetry.) Choose a positive integer $p$ which is relatively prime to $n$ and another positive integer $q$ such that $p q \equiv 1 \bmod n$. We define a paragroup symmetry $\sigma$ by $\sigma(i)=p i \bmod n$ for $i \in \mathbf{Z}_{n}$ and $\sigma(j)=q j \bmod n$ for $j \in \hat{\mathbf{Z}}_{n}$. Then the connection above is invariant under this $\sigma$. We then have flatness automatically, so we get many examples of new principal graphs with integer indices very easily.

Next example of a symmetry fixing $*$ is a $\mathbf{Z}_{2}$-symmetry of $A_{\text {odd }}^{(1)}$ considered in [IK] This is related to classification of subfactors with index 4 . We label vertices of $A_{2 n-5}^{(1)}$ as follows.

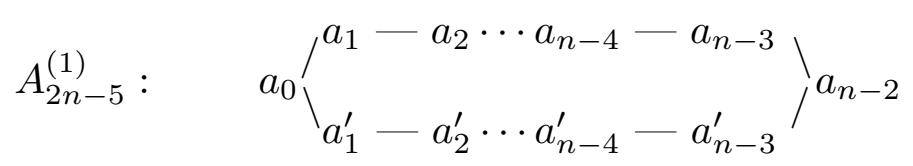

The symmetry $\sigma$ fixed $a_{0}$ and $a_{n-2}$, and switches $a_{i}$ and $a_{i}^{\prime}, 1 \leq i \leq n-3$. There are $n-2$ mutually non-equivalent flat connections on this diagram, and they correspond to locally trivial subfactors with index 4. (So this classification also follows from Connes' classification of automorphisms [Co].) Then we can prove that the connection is chosen so that it is invariant under $\sigma$, then we can get orbifold subfactors from them. Our new principal graph is the following $D_{n}^{(1)}$.

$$
D_{n}^{(1)}: \quad{ }_{b_{0}^{\prime}}^{b_{0}} b_{1}-b_{2} \cdots b_{n-4}-b_{n-3} \backslash_{b_{n-2}^{\prime}}^{b_{n-2}}
$$

For classification of subfactors with the principal graph $D_{n}^{(1)}$, we also have to prove that all of these $n-2$ flat connections are really non-equivalent, and there are nothing more. But these can be proved with the use of the intertwining Yang-Baxter equation for the two graphs as in [IK].

Remark 3.1. S. Popa [P3] announced a complete classification of subfactors with index 4. He gave numbers of conjugacy classes for each possible principal graphs, including infinite graphs $A_{\infty}, A_{\infty, \infty}, D_{\infty}$, but the number was left open only for $D_{n}^{(1)}$. (His classification of subfactors with principal graph $D_{n}^{(1)}$ was given in terms of certain 3rd cohomology group elements and it was hard to compute the number of conjugacy classes.) On the other hand, Ocneanu showed a classification table of subfactors with index 4 in his invited talk at the ICM-90, and in it, he claimed that there is a unique subfactor with a principal graph $D_{n}^{(1)}$. But our method as above easily shows that the 
right number is $n-2$ and Ocneanu's announced uniqueness is invalid, and gives the last missing number in Popa's list.

\section{$\S 4$ Orbifold construction II: $*$ is moved.}

Now we work on more interesting orbifold constructions. These are in duality to the orbifold constructions in $§ 3$. First we consider subfactors with principal graph $D_{n}$. Ocneanu announced in 1987 that there is a unique subfactor for each $D_{2 n}$ and there are no subfactors with the principal graph $D_{2 n+1}$ among his announced classification of subfactors with index less than 4 [O1]. But he has not published his proof. (See Remark 4.2 below.) Here we show that our orbifold method produces a proof of the claim above on $D_{n}$ as in $[\mathrm{Ka}]$.

First note that the graph $A_{2 n-3}$ has a $\mathbf{Z}_{2}$-symmetry $\sigma$. It can be proved that a connection on $A_{2 n-3}$ can be chosen so that it is invariant under $\sigma$. (It is possible to choose a connection to be real, but then this is not a right choice because it is not invariant under $\sigma$.) Now we want to apply the same procedure as in $\S 3$, but we cannot do so immediately, because this $\sigma$ does move the distinguished vertex $*$. That is, we cannot apply $\sigma$ even at the starting algebra $A_{0,0}=\mathbf{C}$ of the double sequence of string algebras. To overcome this difficulty, we modify the construction of the double sequence of string algebras as follows. We allow strings to start one of $*$ and $\sigma(*)$. Multiplication rules and identification based on connections are kept same. In this way, we get a double sequence $\left\{A_{n, m}\right\}$ of string algebras starting from $A_{0,0}=\mathbf{C} \oplus \mathbf{C}$ as in [Ka]. Then, we can apply $\sigma$ to each $A_{m, n}$ and we get another double sequence $A_{m, n}^{\sigma}$. It is not hard to see that this is indeed the double sequence of string algebras of $D_{n}$. Thus the flatness problem of $D_{n}$ is reduced to some computational problem of the connection of $A_{2 n-3}$. That is, as in Theorem $2.1(3)$, we can prove that flatness of the connection on $D_{n}$ is equivalent to the equality that some large partion function has value 1 . Now induction shows that this partition function value is $(-1)^{n}$, which proves Ocneanu's announcement on $D_{n}$. This can be understood as follows in our general settings. If the symmetry fixes the *, then the orbifold construction automatically has flatness as in $\S 3$, but if the symmetry does move the $*$, there arises an obstruction for flatness in the orbifold procedure. This obstruction eliminates $D_{2 n+1}$ but such an obstruction does not exist for $D_{2 n}$.

Furthermore, one can prove that the modification above of the string algebra double sequence construction still gives the same subfactor of type $A_{2 n-3}$. One way to see this is that we can compute the principal graph of this subfactor by Ocneanu's compactness argument [O3]. Then it is easy to see that the principal graph is $A_{2 n-3}$. Another way is that we prove the modified construction gives a subfactor with relative McDuff property. This is done by seeing that the horizontal Jones projections make central sequences in the subfactor for the ambient factor. (See [Bi].) Then it is easy to see that we get a conjugate subfactor by cutting the factors by a projection in the subfactor. (Also see [P1, page 200].) This means that a subfactor with the principal graph $D_{2 n}$ is realized as $N^{\sigma} \subset M^{\sigma}$, where $N \subset M$ has a principal graph $A_{4 n-3}$ and $\sigma$ is an automorphism of $M$ with order 2 fixing $N$ globally. (Note that the orbifold construction as in $\S 3$ for $D_{n}$ and Takesaki duality easily produce the following: If $D_{n}$ principal graph is realized, the subfactor is of the above form of the simultaneous fixed point algebra of the $A_{2 n-3}$ subfactor. Thus the difference between $D_{2 n}$ and $D_{2 n+1}$ comes from the difference between $A_{4 n-3}$ and $A_{4 n-1}$.) 
Remark 4.1. Impossibility of $D_{2 n+1}$ as a principal graph has also been independently proved by [I1], [SV]. Their method is to prove inconsistency of decomposition rules of multiplication of endomorphisms or bimodules for $D_{2 n+1}$. This method was also claimed by Ocneanu without a proof. For impossibility of $E_{7}$, the author verified non-flatness by a computer $[\mathrm{Ka}]$, and inconsistency of decomposition rules was verified by [SV], as Ocneanu announced. Izumi [I1] gave a much simpler proof just by looking at the Perron-Frobenius eigenvector entry. (This was essentially in [P2, Theorem 3.8].) For $E_{6}$, Bion-Nadal [BN] gave a construction, and then Ocneanu's general machinery easily produces that there are two and only two subfactors for the $E_{6}$ principal graph [O1, $\mathrm{O} 3, \mathrm{Ka}]$. For $E_{8}$, Izumi [I2] checked flatness and thus proved that there are two and only two subfactors for the $E_{8}$ principal graph. In this way, we now have a complete proof of classification of subfactors with index less than 4 announced by Ocneanu.

U. Haagerup said to the author that he has also verified this classification by his method based on bimodules.

Remark 4.2. A. Ocneanu sketched his original proof of flatness of $D_{2 n}$ in his lectures at Tokyo in July of 1990 [O3]. But the details were not clear and the author could not complete the proof along this line, so the author found a different proof based on orbifold method in December of 1990 [Ka] as described above. Then in October of 1991, A. Ocneanu showed further details to the author on his original method and the author understood his complete proof. We include his arguments in Appendix here because his method is quite different from ours, and is of another interest. His proof is somewhat shorter than ours, but it does not give a realization of $D_{2 n}$ as a simultaneous fixed point algebra of $A_{4 n-3}$ and it seems difficult to extend this method to the Hecke algebra settings while ours does as below. The author thanks Prof. A. Ocneanu for showing this proof and permitting us to include it here.

H. Wenzl constructed his Hecke algebra subfactors with index values $\frac{\sin ^{2}(N \pi / k)}{\sin ^{2}(\pi / k)}$ as a natural generalization of Jones' subfactors of type $A_{n}$ with index values $4 \cos ^{2} \frac{\pi}{n+1}$ in [We]. It turned out that these subfactors correspond to 2-variable link invariant (HOMFLY) polynomial [FYHLMO], quantum groups $U_{q}\left(s l_{N}\right)$, and the Jimbo-MiwaOkado solutions to the Yang-Baxter equation [JMO1, JMO2]. Now we show that we can also apply the orbifold construction to Wenzl's Hecke algebra subfactors [EK].

In order to apply the orbifold method, we have to know the paragroups of the Hecke algebra subfactors. Wenzl computed the principal graphs of his subfactors in an unpublished work but could not obtain paragroups. We compute the paragroups with the Yang-Baxter equation in [EK]. Because Wenzl's representation is a certain trigonometric limit of Boltzmann weights of Jimbo-Miwa-Okado [JMO1, JMO2] in the form of elliptic functions, we still have the Yang-Baxter equation. Roche's result $[R]$ implies that the face operators are flat when we have the Yang-Baxter equation, and now the Hecke algebra generators are essentially face operators. This is a typical example that the Yang-Baxter equation implies flatness. (But in general, the subfactor does not have good series of generators and thus the Yang-Baxter equation does not produce full flatness.) Furthermore, Wenzl's construction of subfactor uses commuting squares of period $N$, but the canonical form arising as higher relative commutants should have a 
period 2. This means that the construction of Wenzl's Hecke algebra subfactor is not in the canonical form in the sesne of Popa and Ocneanu. But by the use of the YangBaxter equation as above and the crossing symmetry, we can compute the paragroup and modify the construction so that it has a period 2 .

Here we show how to proceed in the case $N=3$ for the orbifold construction. Then first we have to modify the double sequence again so that it now starts with $A_{0,0}=\mathbf{C} \oplus \mathbf{C} \oplus \mathbf{C}$. Then we can define a symmetry $\sigma$ of order 3 and make a fixed point algebra $A_{m, n}^{\sigma}$. Again, flatness for orbifolds can be reduced to computations of certain partition functions of the original connection. By the Yang-Baxter equation, we can compute the values. In the case $D_{n}$, there was a difference between $D_{2 n}$ and $D_{2 n+1}$, but now it turns out that an obstruction for flatness vanishes in all the cases. It appears that this difference between $N=2$ and $N=3$ comes from parity of $N$. Because Wenzl's subfactors are regarded as a generalization of the $A_{n}$-sequence, our construction gives a "generalized $D_{n}$ " sequence. (Graphs $\mathcal{D}$ were considered in $[\mathrm{FG}]$ as a generalization of $D_{n}$-sequence, but our principal graph is only a part of their graphs.) For some examples of our principal graphs, see [EK].

Appendix: Ocneanu's original proof of flatness of $D_{2 n}$

Here we include an outline of Ocneanu's original proof of flatness of $D_{2 n}$ along the line suggested in [O3]. The author thanks him for showing the arguments and permitting him to include this here. We did not try to supply all the computational details, but readers familiar with $[\mathrm{O} 3, \mathrm{Ka}]$ should have no difficulty in understanding.

We identify $\mathcal{G}$ and $\mathcal{H}$ and label some vertices of it as follows.

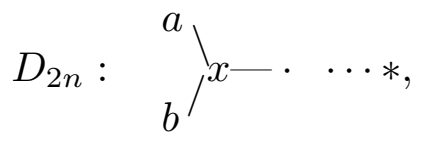

Define a connection on this diagram as follows. We start from $*$, and until the vertex $x$, we choose a real connection as in the case of diagrams $A_{n}$. (See [O3], for example.) At the vertex $x$, we have $3 \times 3$-unitary matrix as follows.

$$
\left(\begin{array}{lll}
c_{11} & c_{12} & c_{13} \\
c_{21} & c_{22} & c_{23} \\
c_{31} & c_{32} & c_{33}
\end{array}\right),
$$

where columns are labeled by $\cdot, a, b$ from the left to the right and rows are labeled by $\cdot, a, b$ from the top to the bottom. We choose the connection so that $c_{11}, c_{12}, c_{13}, c_{21}, c_{31} \in \mathbf{R}$ and $c_{22}=c_{33}=\bar{c}_{23}=\bar{c}_{32}$. (This is possible. See [O3, IV.2] and [Ka, §3]. Note that this choice of gauges is different from the standard one in [O1, O3, Ka].) We fix this connection. Note that this connection is invariant under the graph automorphism $\sigma$ flipping $a$ and $b$.

We make a double sequence of string algebras starting from $*$. Denote by $q, q^{\prime}$ horizontal strings $(*-\cdots-x-a, *-\cdots-x-a)$ and $(*-\cdots-x-b, *-\cdots-x-b)$ respectively. (Each string has length $2 n-2$.) We also denote by $p, p^{\prime}$ the vertical strings with the same expressions. Note that $p+p^{\prime}$ is a flat projection orthogonal to the vertical Jones projections $e_{1}, e_{2}, \cdots, e_{2 n-3}$. Then $q\left(p+p^{\prime}\right) q$ can be identified with a string with length $2 n-2$ starting from $a$. We can express this as a sume of mutually orthogonal 
projections by decomposing it according to the endpoints of strings. Then we have the following.

Claim. For each n, one of the following holds under the situation above.

(1) If the endpoint for $q\left(p+p^{\prime}\right) q$ is a, then the corresponding projection is 0 and if the endpoints for $q\left(p+p^{\prime}\right) q$ is not a, then the corresponding projection is of rank 1 .

(2) If the endpoint for $q\left(p+p^{\prime}\right) q$ is $b$, then the corresponding projection is 0 and if the endpoints for $q\left(p+p^{\prime}\right) q$ is not $b$, then the corresponding projection is of rank 1 .

Furthermore, we have the same claim for $q^{\prime}\left(p+p^{\prime}\right) q^{\prime}$, and if we have (1) [resp. (2)] for $q\left(p+p^{\prime}\right) q$, then we have (2) [resp. (1)] for $q^{\prime}\left(p+p^{\prime}\right) q^{\prime}$. This claim is proved by looking at the embeddings of the Jones projections into the string algebra of $D_{2 n}$ starting from $a$ or $b$. (See [GHJ], [Ok, page 99].)

Now it is enough to prove

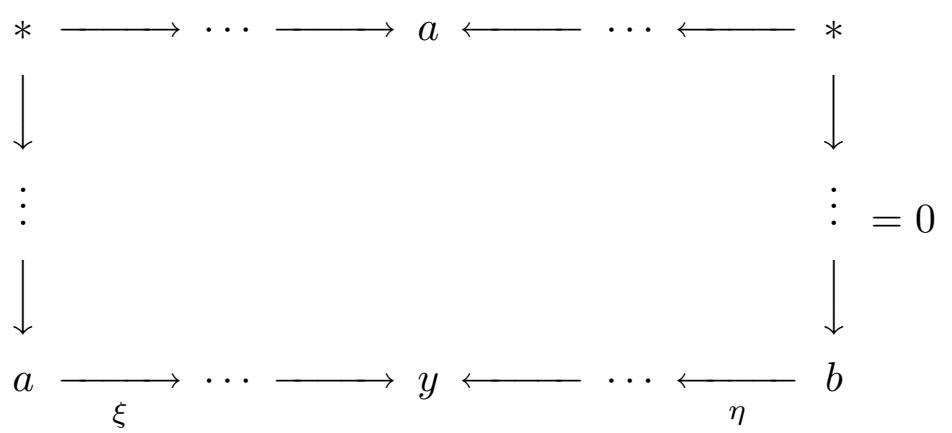

for all the $\xi, \eta, y$, where the diagram above is of size $(2 n-2) \times 2(2 n-2)$. We prove this as follows.

If $y=a$ or $y=b$, then the claim above proves the desired equality. Suppose $y \neq a, b$. Again by the claim above, it is enough to prove the equality above for a single $\sigma$ and $\eta=\sigma(\xi)$ after a certain change of basis in the path Hilbert space. Because $q+q^{\prime}$ is flat, and the connection is $\sigma$-invariant, we get the following identities.

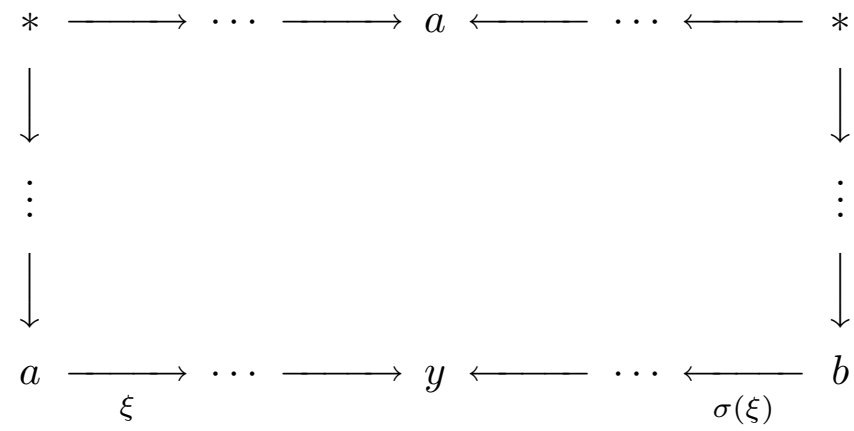




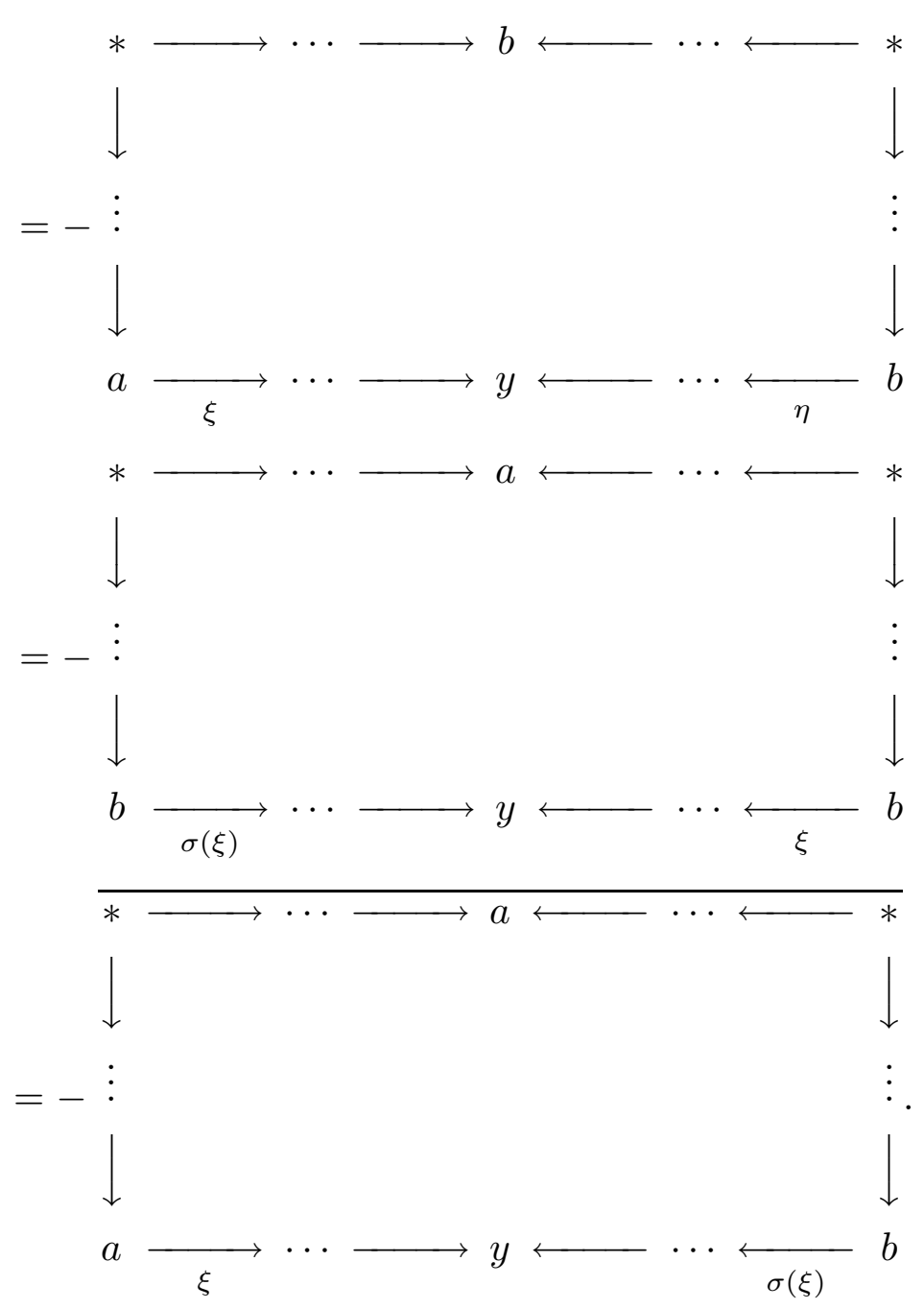

This implies that

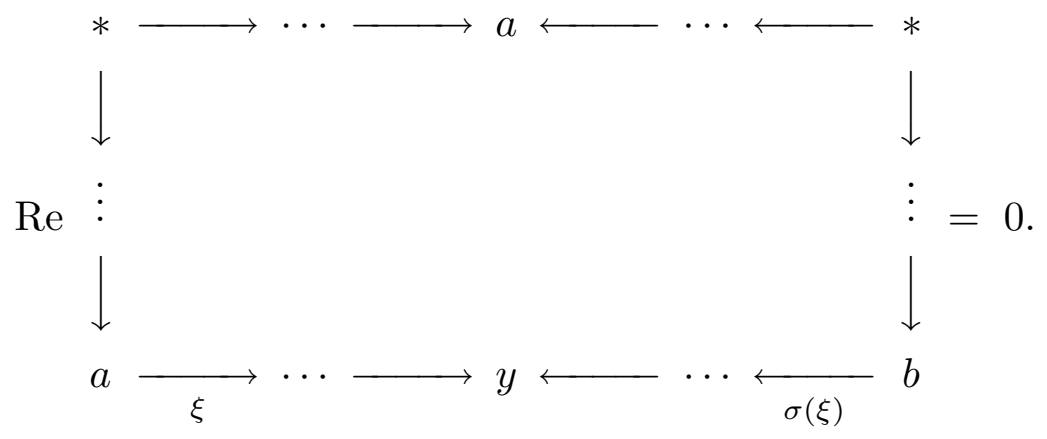

Now the following claim finishes the proof.

Claim. Each $2 \times 2$-diagram with even vertices of $D_{2 n}$ on the boundary has a real value.

This claim is checked directly. 


\section{REFERENCES}

[Ba] R. J. Baxter, "Exactly solved models in statistical mechanics", Academic Press, New York, 1982.

[BN] J. Bion-Nadal, An example of a subfactor of the hyperfinite $I_{1}$ factor whose principal graph invariant is the Coxeter graph $E_{6}$, in "Current Topics in Operator Algebras", World Scientific Publishing, 1991, pp. 104-113.

[Bi] D. Bisch, On the existence of central sequences in subfactors, Trans. Amer. Math. Soc. 321 (1990), 117-128.

[BEEK1] O. Bratteli, G. A. Elliott, D. E. Evans, \& A. Kishimoto, Non-commutative spheres, Inter. J. Math. 2 (1991), 139-166.

[BEEK2] O. Bratteli, G. A. Elliott, D. E. Evans, \& A. Kishimoto, Finite group actions on AF algebras obtained by folding the interval, (to appear in K-theory).

[BEEK3] O. Bratteli, G. A. Elliott, D. E. Evans, \& A. Kishimoto, Non-commutative spheres II: rational rotations, (to appear in J. Operator Theory).

[BEK] O. Bratteli, D. E. Evans, \& A. Kishimoto, Crossed products of totally disconnected spaces by $\mathbf{Z}_{2} * \mathbf{Z}_{2}$, preprint, Swansea, 1991.

[BK] O. Bratteli \& A. Kishimoto, Non-commutative spheres III: irrational rotations, preprint, Trondheim, 1991.

[Ch] M. Choda, Duality for finite bipartite graphs, to appear in Pac. J. Math.

[Co] A. Connes, Periodic automorphisms of the hyperfinite factor of type $I_{1}$, Acta Sci. Math. 39, 39-66 (1977).

[DJMO] E. Date, M. Jimbo, T. Miwa, \& M. Okado, Solvable lattice models, in "Theta functions - Bowdoin 1987, Part 1," Proc. Sympos. Pure Math. Vol. 49, Amer. Math. Soc., Providence, R.I., pp. 295-332.

[DZ] P. Di Francesco \& J.-B. Zuber, $S U(N)$ lattice integrable models associated with graphs, Nucl. Phys. B338 (1990), 602-646.

[E1] D. E. Evans, The $C^{*}$-algebras of topological Markov chains, Tokyo Metropolitan University Lecture Notes, 1984.

[E2] D. E. Evans, Quasi-product states on $C^{*}$-algebras, in "Operator algebras and their connections with topology and ergodic theory", Springer Lecture Notes in Math., 1132 (1985), 129-151.

[EG] D. E. Evans \& J. D. Gould, Embeddings and dimension groups of non-commutative AF algebras associated to models in classical statistical mechanics, preprint, Swansea.

[EK] D. E. Evans \& Y. Kawahigashi, Orbifold subfactors from Hecke algebras, preprint. [FG] P. Fendley \& P. Ginsparg, Non-critical orbifolds, Nucl. Phys. B324 (1989), 549580.

[FYHLMO] P. Freyd, D. Yetter; J. Hoste; W. Lickorish, K. Millet; \& A. Ocneanu, A new polynomial invariant of knots and links, Bull. Amer. Math. Soc. 12 (1985), 239-246.

[GHJ] F. Goodman, P. de la Harpe, \& V. F. R. Jones, "Coxeter graphs and towers of algebras", MSRI publications 14, Springer, 1989.

[HS] U. Haagerup \& J. Schou, in preparation.

[H] U. Haagerup, in preparation.

[I1] M. Izumi, Application of fusion rules to classification of subfactors, to appear in Publ. RIMS Kyoto Univ.

[I2] M. Izumi, On flatness of the Coxeter graph $E_{8}$, preprint. 
[IK] M. Izumi \& Y. Kawahigashi, Classification of subfactors with the principal graph $D_{n}^{(1)}$, to appear in J. Funct. Anal.

[Ji] M. Jimbo (editor), "Yang-Baxter equation in integrable systems", Advanced Series in Mathematical Physics, Vol. 10, World Scientific, 1989.

[JMO1] M. Jimbo, T, Miwa, \& M. Okado, Solvable lattice models whose states are dominant integral weights of $A_{n-1}^{(1)}$, Lett. Math. Phys. 14 (1987), 123-131.

[JMO2] M. Jimbo, T, Miwa, \& M. Okado, Solvable lattice models related to the vector representation of classical simple Lie algebras, Comm. Math. Phys. 116 (1988), 507-525.

[J] V. F. R. Jones, Index for subfactors, Invent. Math. 72 (1983), 1-15.

[Ka] Y. Kawahigashi, On flatness of Ocneanu's connections on the Dynkin diagrams and classification of subfactors, University of Tokyo, preprint, 1990.

[Ko] I. Kostov, Free field presentation of the $A_{n}$ coset models on the torus, Nucl. Phys. B300 (1988), 559-587.

[L1] P. Loi, On automorphisms of subfactors, preprint.

[L2] P. Loi, On the derived towers of certain inclusions of type $I_{\lambda} I_{\lambda}$ factors of index 4 , preprint.

[O1] A. Ocneanu, Quantized group string algebras and Galois theory for algebras, in "Operator algebras and applications, Vol. 2 (Warwick, 1987)," London Math. Soc. Lect.

Note Series Vol. 136, Cambridge University Press, 1988, pp. 119-172.

[O2] A. Ocneanu, Graph geometry, quantized groups and nonamenable subfactors, Lake Tahoe Lectures, June-July, 1989.

[O3] A. Ocneanu, "Quantum symmetry, differential geometry of finite graphs and classification of subfactors", University of Tokyo Seminary Notes 45, (Notes recorded by Y. Kawahigashi), 1991.

[O4] A. Ocneanu, in preparation.

[Ok] S. Okamoto, Invariants for subfactors arising from Coxeter graphs, in "Current Topics in Operator Algebras", World Scientific Publishing, 1991, pp. 84-103.

[PP] M. Pimsner \& S. Popa, Iterating the basic constructions, Trans. Amer. Math. Soc. 310 (1988), 127-134.

[P1] S. Popa, Relative dimension, towers of projections and commuting squares of subfactors, Pac. J. Math. 137 (1989), 181-207.

[P2] S. Popa, Classification of subfactors: reduction to commuting squares, Invent. Math. 101 (1990), 19-43.

[P3] S. Popa, Sur la classification des sousfacteurs d'indice fini du facteur hyperfini, C. R. Acad. Sc. Paris. 311 (1990), 95-100.

[R] Ph. Roche, Ocneanu cell calculus and integrable lattice models, Comm. Math. Phys. 127 (1990), 395-424.

[Sc] J. Schou, Commuting squares and index for subfactors, Ph.D. thesis, Odense University, 1990.

[Su] V.S. Sunder, A model for AF-algebras and a representation of the Jones projections, J. Operator Theory 18 (1987), 289-301.

[SV] V. S. Sunder \& A. K. Vijayarajan, On the non-occurrence of the Coxeter graphs $E_{7}, D_{2 n+1}$ as principal graphs of an inclusion of $I I_{1}$ factors, preprint.

[T] M. Takesaki, "Theory of Operator Algebras I", Springer, New York, 1979.

[TV] V. G. Turaev \& O. Y. Viro, State sum invariants of 3-manifolds and quantum $6 j$-symbols, preprint. 
[Wa] A. J. Wassermann, Coactions and Yang-Baxter equations for ergodic actions and subfactors, in "Operator algebras and applications, Vol. 2 (Warwick, 1987)," London Math. Soc. Lect. Note Series Vol. 136, Cambridge University Press, 1988, pp. 203-236. [We] H. Wenzl, Hecke algebras of type A and subfactors, Invent. Math. 92 (1988), 345383.

[Y] S. Yamagami, A report on Ocneanu's lecture, preprint. 